\title{
Preface \\ Systemic sclerosis and related connective tissue diseases: present and future
}

\author{
Lewis J Rubin ${ }^{1}$, Carol M Black², Christopher P Denton² and James R Seibold ${ }^{3}$
}

\begin{abstract}
1'Division of Pulmonary and Critical Care Medicine, University of California, San Diego, Campus Point Drive, La Jolla, California 92037-7381, USA
${ }^{2}$ Centre for Rheumatology, Royal Free and University College Medical School, Rowland Hill Street, London, NW3 2PF, UK

3 University of Michigan Scleroderma Program, East Medical Center Drive, Ann Arbor, Michigan 48109-0358, USA
\end{abstract}

Corresponding author: Lewis J Rubin, ljrubin@ucsd.edu

Published: 15 August 2007

This article is online at http://arthritis-research.com/content/9/S2/S1

(c) 2007 BioMed Central Ltd
Arthritis Research \& Therapy 2007, 9(Suppl 2):S1 (doi:10.1186/ar2185)

many CTDs. While our knowledge on the role played by angiogenesis in various CTDs has advanced significantly, Koch and Distler point out that there are many unanswered questions that must be addressed if we are to elucidate fully the pathogenesis of SSc and improve its management. The critical question is whether it will be therapeutically useful to augment angiogenesis and whether it will be possible to manipulate selectively the positive and negative effects of mediators such as ET-1.

Fibrosis, caused by excessive extracellular matrix accumulation, is a unifying feature of many CTDs and is a cardinal pathologic event in SSc. Krieg, Abraham, and Lafyatis [3] review a series of experimental studies that suggest that a complex network of intercellular interactions involving keratinocytes, fibroblasts, and an array of molecular mediators drive the pathogenic events that lead to fibrosis.

Our understanding of the genetic factors that may play a role in SSc has increased considerably in recent years; genes have a role to play in either determining susceptibility or influencing the phenotypic expression of the disease, or both. The article by Mayes and Trojanowska [4] reviews the genetic loci that appear to be associated with SSc. Genetic studies are considerably hampered by the complexity of SSc disease. Mayes and Trojanowska review recent evidence for the contribution of a candidate gene, Fli1, whose dysregulation appears to play a pathologic role in SSc skin fibrosis as well as having an effect on vessel degeneration.

The next article, by Denton [5], reviews therapeutic targets in SSc, their links to pathology, and the importance of crosstalk between mediators. The relevance of the timing of intervention to possible outcomes of therapeutic interventions is discussed. Strategies for disease modification and novel

$\mathrm{CTD}=$ connective tissue disease $; \mathrm{ET}=$ endothelin; $\mathrm{PH}=$ pulmonary hypertension; $\mathrm{SSc}=$ systemic sclerosis. 
therapeutic targets are also presented. The lack of clinical success from targeting logical profibrotic mediators is contrasted with positive results on vasculopathic manifestations of CTD with dual endothelin receptor antagonism.

Matucci-Cerinic, Steen, Furst, and Seibold [6] review data from recent clinical trials and information derived from retrospective observational studies from databases and patient registries. They also discuss how we may be able to use this information to provide the best possible outcomes for patients with SSc. The wealth of clinical information has improved our understanding of the diverse clinical course of the disease. Furthermore, these data can be used to refine existing outcome measures for the design of future clinical trials that maximize the likelihood of observing a positive treatment effect with the drugs at our disposal. The authors go on to discuss the results of trials in interstitial lung disease, which underscore the need for a definitive trial design template for studies in this patient population. In a progressive disease such as SSc, it is becoming increasingly clear that the greatest chance of demonstrating a diseasemodifying effect is to treat aggressively and early in the disease process. A key challenge for the future is to identify risk factors that will allow enrichment of clinical trial patient populations, such that clinically relevant treatment effects can be observed.

In his article, Baughman [7] reviews the pathophysiology of pulmonary hypertension $(\mathrm{PH})$ associated with sarcoidosis, the role of ET-1, and the rationale behind ET-1 blockade. There is mounting evidence to suggest that $\mathrm{PH}$ is a relatively common complication of sarcoidosis, not only in patients with end-stage pulmonary fibrosis (in whom it is predictive of increased mortality) but also in those with earlier stage pulmonary disease. Increased levels of ET-1 in plasma and bronchoalveolar lavage of some sarcoid patients are consistent with a pathogenic role in both pulmonary fibrosis and sarcoidosis associated $\mathrm{PH}$. Preliminary clinical data with endothelin receptor antagonists in sarcoidosis are encouraging and, in combination with anti-inflammatory medications, these agents may provide additional clinical benefit and improve outcomes in patients with sarcoidosis associated $\mathrm{PH}$.

The next article, by Guillevin and Dörner [8], focuses on systemic vasculitis, which is a rare and destructive inflammatory disease of blood vessel walls that occurs in all CTDs. This inflammatory activity is believed to contribute to the increased risk for cardiovascular morbidity and mortality in rheumatoid arthritis and to the accelerated atherosclerosis seen in systemic lupus erythematosus. Endothelial cell activation via autoantibody or immune complex binding is a common pathogenic pathway in the systemic vasculitis associated with both rheumatoid arthritis and systemic lupus erythematosus, and there is evidence that ET- 1 plays a role in some clinical manifestations of vasculitis. Therefore, endothelin receptor antagonism may be of clinical benefit in the treatment of specific vascular manifestations of vasculitis as an adjunct to standard therapy with glucocorticoids and immunosuppressant agents.

In their concluding article, Rubin, Black, Denton, and Seibold discuss future directions in the areas of SSc research and targets for therapy [9].

In summary, the collection of articles included in this supplement provides a review of our current understanding of SSc and other CTDs, the pathophysiologic processes involved, and the approach to management. Although considerable progress has been made in recent years, we have a long way to go. Early diagnosis and treatment with effective therapies are key to providing patients with the best possible long-term outcomes.

\section{Competing interests}

All of the authors have been investigators and consultants for Actelion Pharmaceuticals Ltd.

\section{Acknowledgements}

The authors would like to acknowledge medical writing support funded by an educational grant from Actelion Pharmaceuticals Ltd.

This article is part of Arthritis Research \& Therapy Volume 9 Supplement 2: Advances in systemic sclerosis and related fibrotic and vascular conditions, and is based on presentations made at a symposium entitled Advances in systemic sclerosis and connective tissue disease, sponsored by Actelion Pharmaceuticals Ltd, held in Athens, Greece in April 2006. The full contents of the supplement are available online at http://arthritis-research.com/ supplements/9/S2. This supplement has been supported by an educational grant from Actelion Pharmaceuticals Ltd.

\section{References}

1. Abraham D, Distler O: How does endothelial cell injury start? The role of endothelin in systemic sclerosis. Arthritis Res Ther 2007, 9(Suppl 2):S2.

2. Koch $A E$, Distler $\mathrm{O}$ : Vasculopathy and disordered angiogenesis in selected rheumatic diseases: rheumatoid arthritis and systemic sclerosis. Arthritis Res Ther 2007, 9(Suppl 2):S3.

3. Krieg T, Abraham D, Lafyatis R: Fibrosis in connective tissue disease: the role of the myofibroblast and fibroblast-epithelial cell interactions. Arthritis Res Ther 2007, 9(Suppl 2):S4.

4. Mayes MD, Trojanowska T: Genetic factors in systemic sclerosis. Arthritis Res Ther 2007, 9(Suppl 2):S5.

5. Denton CP: Therapeutic targets in systemic sclerosis. Arthritis Res Ther 2007, 9(Suppl 2):S6.

6. Matucci-Cerinic M, Steen VD, Furst DE, Seibold JR: Clinical trials in systemic sclerosis: lessons learned and outcomes. Arthritis Res Ther 2007, 9(Suppl 2):S7.

7. Baughman RP: Pulmonary hypertension associated with sarcoidosis. Arthritis Res Ther 2007, 9(Suppl 2):S8.

8. Guillevin L, Dörner T: Vasculitis: mechanisms involved and clinical manifestations. Arthritis Res Ther 2007, 9(Suppl 2):S9.

9. Rubin LJ, Black CM, Denton CP, Seibold JR: Clinical trials and basic research: defining mechanisms and improving treatment in connective tissue disease. Arthritis Res Ther 2007, 9 (Suppl 2):S10. 